Journal for ImmunoTherapy of Cancer

\section{Evaluating the efficacy of a priming dose of cyclophosphamide prior to pembrolizumab to treat metastatic triple negative breast cancer}

To cite: Anders CK, Woodcock MG, Van Swearingen AED, et al. Evaluating the efficacy of a priming dose of cyclophosphamide prior to pembrolizumab to treat metastatic triple negative breast cancer. Journal for ImmunoTherapy of Cancer 2022;10:e003427. doi:10.1136/ jitc-2021-003427

- Additional supplemental material is published online only. To view, please visit the journal online (http://dx.doi.org/10. 1136/jitc-2021-003427).

CKA and MGW contributed equally.

BGV and JSS contributed equally.

Accepted 16 November 2021

Check for updates

(C) Author(s) (or their employer(s)) 2022. Re-use permitted under CC BY-NC. No commercial re-use. See rights and permissions. Published by BMJ.

For numbered affiliations see end of article.

Correspondence to Dr Jonathan S Serody; jonathan_serody@med.unc.edu

\section{ABSTRACT}

Purpose Triple negative breast cancer (TNBC) is characterized by the presence of immune cells in the tumor microenvironment, however, the response to singleagent immune checkpoint inhibitor (ICl) therapy is modest. Preclinical models have demonstrated that intratumoral regulatory $T$ cells $\left(T_{\text {regs }}\right)$ dampen the antitumor response to ICI. We performed a single-arm phase II trial to evaluate the efficacy of a single low dose of cyclophosphamide (Cy) to deplete $\mathrm{T}_{\text {regs }}$ administered before initiating pembrolizumab. Patients and methods 40 patients with pretreated metastatic TNBC were enrolled. The primary endpoints were progression-free survival (PFS) and change in peripheral blood $T_{\text {regs }}$ after Cy. Secondary endpoints included overall response rate (ORR), duration of response, overall survival, treatment-related adverse events (AEs), and correlative evaluations.

Results Median PFS was 1.8 months, and the ORR was $21 \%$. $\mathrm{T}_{\text {regs }}$ were not significantly decreased after Cy prior to ICI $(-3.3 \%, p=0.19)$, and increased significantly after the first cycle of therapy $(+21 \%$ between cycles 1 and 2 , $\mathrm{p}=0.005)$. Immune-related AEs were similar to historical pembrolizumab monotherapy, and were associated with response to therapy $(p=0.02)$. Patients with pretreatment tumors harboring increased expression of $B$ cell metagene signatures and increased circulating $B$ cell receptor repertoire diversity were associated with clinical response and immune-related toxicity (IRT).

Conclusions Among patients with heavily pretreated TNBC, Cy prior to pembrolizumab did not significantly deplete $T_{\text {regs }}$, and in those with decreased numbers there was rapid recovery following therapy. Increased B cell gene expression in baseline samples was associated with clinical response and IRT.

\section{INTRODUCTION}

Triple negative breast cancer (TNBC) lacks expression of the estrogen receptor (ER), progesterone receptor (PR), and human epidermal growth factor receptor 2 (HER2). TNBC is the most aggressive breast cancer subtype and portends a poor prognosis despite initial responses to cytotoxic chemotherapy. ${ }^{1-5}$ TNBC is associated with early and visceral recurrence, and frequently metastasizes to distant sites, particularly to the lungs and brain. ${ }^{16}$ The median progression-free survival (PFS) for patients with metastatic TNBC (mTNBC) with first-line chemotherapy is $<6$ months, and overall survival (OS) is typically $<2$ years from initial metastatic diagnosis. 78

Previously, our group had shown that TNBC was the breast cancer subtype most heavily infiltrated with immune cells. ${ }^{9}$ Despite these findings, the activity of immune checkpoint inhibitor (ICI) that inhibit the PD-1/ PD-L1/2 axis as a monotherapy for TNBC has been relatively modest. ${ }^{10}$ The KEYNOTE-012 study, a phase $1 \mathrm{~b}$ trial of pembrolizumab in heavily pretreated patients with mTNBC, reported an overall response rate (ORR) of $18.5 \%$, with patients progressing at a mean of 3 months post-therapy. ${ }^{11}$ Similar studies evaluating the function of antibodies targeting PD-1 or PD-L1 as a monotherapy have shown response rates of $8 \%-15 \% .{ }^{10}$ More recently, pembrolizumab was approved for patients with mTNBC whose tumors expressed PD-L1 when given with either nanoparticle albuminbound paclitaxel, gemcitabine or carboplatin. In patients with tumors that express PD-L1 $\geq 10$ by the combined positive score, ${ }^{12}$ median PFS was 9.7 months in those getting 
chemotherapy with pembrolizumab compared with 5.6 months for those getting placebo and chemotherapy (HR $0.6595 \%$ CI 0.49 to $0.86 ; \mathrm{p}=0.0012$ ).

The exact mechanism underlying the limited response of patients with TNBC to ICI monotherapy treatment is not well understood. Our group and others have characterized the presence of immunosuppressive cells such as $\mathrm{CD}_{25} 5^{+}$FOXP3-expressing regulatory $\mathrm{T}$ cells $\left(\mathrm{T}_{\text {regs }}\right)$, M2-like macrophages and myeloid-derived suppressor cells in the TNBC tumor microenvironment (TME) that may contribute to resistance mechanisms ${ }^{13-18}$ Previously, we had shown that the depletion of intratumoral $\mathrm{T}_{\text {regs }}$ enhanced the anti-tumor response in genetic murine models of TNBC treated with dual ICI therapy. ${ }^{19}$ In these preclinical studies, treatment with low-dose cyclophosphamide $(\mathrm{Cy})$ reduced $\mathrm{T}_{\text {reg }}$ numbers in tumors, suggesting a method of sensitizing TNBC to ICI. ${ }^{19}$

Based on our preclinical observations, we initiated a multicenter, single-arm phase II study (NCT02768701) to evaluate the safety and activity of a single low dose of $\mathrm{Cy}$ to deplete $\mathrm{T}_{\text {regs }}$ prior to pembrolizumab in patients with previously treated mTNBC. Primary objectives were the assessment of PFS and the ability of low-dose Cy to deplete peripheral blood $\mathrm{T}_{\text {regs }}$. We also report the ORR, duration of response (DoR), OS, treatment-related adverse effects (AEs), and correlative science objectives using RNA-seq, whole exome sequencing (WES) and adaptive immune receptor repertoire profiling of tumor and peripheral blood from patients enrolled in this study to evaluate if we could confirm a critical role for B cells in the immune response to anti-PD-1 mAb in patients with breast cancer.

\section{MATERIALS AND METHODS Patients}

Patients were enrolled at five institutions (UNC-Chapel Hill, University of Pittsburgh, Moses Cone, UNC-Rex Cancer Center, and George Washington University) from November, 2016 to February, 2018. Eligible patients had histologically confirmed mTNBC (ER and PR negative, $<1 \%$; HER2 negative, $0-1$ by immunohistochemistry or non-amplified by fluorescence in situ hybridization). Eligible patients had received at least one prior cytotoxic treatment in the metastatic setting; stable brain metastases were allowed. Additional inclusion criteria included Eastern Cooperative Oncology Group (ECOG) performance status of $\leq 1$, adequate organ function, and having been off corticosteroids for at least 7 days prior to entry.

Patients were excluded if they were pregnant or breast feeding, had a diagnosis of immunodeficiency or need for ongoing steroid therapy, receipt of prior immunotherapy, a history of HIV or active hepatitis B/C, or had progressive brain or leptomeningeal metastases. All patients provided written informed consent.

\section{Study design}

This was an open-label, single-arm phase II study. The primary endpoints were PFS and change in peripheral blood $\mathrm{T}_{\text {regs }}$ after a single dose of Cy. Secondary endpoints were ORR, DoR, OS, treatment-related AEs, and correlative science objectives.

\section{Administration of study treatment}

Enrolled patients received a single priming dose of 300 $\mathrm{mg} / \mathrm{m}^{2}$ Cy intravenously on cycle 1 , day 1 (C1D1). The next day (C1D2), patients received $200 \mathrm{mg}$ pembrolizumab intravenously. Pembrolizumab was given every 3 weeks until either progressive disease (PD) was seen or the patient suffered significant toxicity, at which point they discontinued protocol-directed therapy.

\section{Efficacy assessments}

Serial CT scans of the chest, abdomen, and pelvis, and a nuclear bone scan to evaluate disease burden were obtained at 8 weeks, and then every 8-12 weeks thereafter. Response criteria was evaluated using modified Response Evaluation Criteria In Solid Tumors V.1.1 (RECIST V.1.1 $)^{20}$ for the primary objective of PFS, and the best response was determined for each patient. A complete response (CR), partial response (PR), stable disease (SD) and PD were defined as in RECIST V.1.1. SD did not meet the criteria either PR or PD. ORR was defined as CR +PR. Clinical benefit (CBR) was defined as $\mathrm{CR}+\mathrm{PR}+\mathrm{SD}$ for at least 6 months from the best response date. Adverse events (AEs) were graded according to the NCI Common Toxicity Criteria for Adverse Events (NCI CTCAE), V.4.0. DOR was defined as time from determination of treatment response to progression. AEs were assessed every 3 weeks and graded according to the NCI CTCAE v4.0; a duration of an $\mathrm{AE}$ was not required. Immune-related toxicity (IRT) was defined as those AEs in common with immune-related AEs previously reported with single agent pembrolizumab, specifically colitis, xerostomia, fever, influenza-like symptoms, hypothyroidism, immune system disorders, pruritus, or rash. ${ }^{11}{ }^{21}$ IRT was evaluated using CTCAE V.4.0, with any grade considered a toxicity regardless of duration.

\section{Peripheral blood $\mathrm{T}_{\text {reg }}$ levels}

Peripheral blood was collected on C1D1 prior to $\mathrm{Cy}$ administration, C1D2 prior to pembrolizumab, and on D1 of each subsequent cycle of pembrolizumab. Peripheral blood mononuclear cells (PBMCs) were isolated from peripheral blood via Ficol gradient and cryopreserved in $10 \%$ DMSO and $90 \%$ fetal bovine serum. Cryopreserved PBMCs were subsequently thawed, washed free of DMSO and exogenous protein with Hanks Balanced Salt Solution, HBSS, (Gibco) and concentrated to $1-5 \times 106 / \mathrm{mL}$. Cells were first incubated with Live/Dead Aqua (Molecular Probes L34066) for $30 \mathrm{~min}$ at room temperature in the dark before washing with $0.1 \%$ sodium azide (Sigma) and $0.5 \%$ Human Serum Albumin (HSA) (CSL Behring). Purified murine IgG (Sigma) was added to a final concentration of 200 micrograms $/ \mathrm{ml}$ to block non-specific binding of the fluorescently labeled murine monoclonal antibodies. Fluorescently labeled antibodies were added 
to appropriate tubes (including FMO controls) and incubated on ice for $30 \mathrm{~min}$ in the dark. The following antibodies were used at predetermined antibody concentrations: CD 19- APC-Cy7 (BD 557791) and CD14- APCCy7 (BD 561709) were used in a dump channel to exclude B lymphocytes and monocytes respectively from the analyses, CD45-V450 (BD Biosciences 560367), CD4FITC (BD Pharmingen 561005), CD8-PerCP-Cy5 (BD Biosciences 560662), CD25-PE (BD Biosciences 341010), CD127-PE-Cy7 (BD Biosciences 560822). Cells were incubated with antibodies on ice for $30 \mathrm{~min}$ in the dark. After incubation, the cells were washed twice in FACS Staining Buffer (.1\% sodium azide, $0.5 \%$ HSA in HBSS) and resuspended in FoxP3 buffer A for $10 \mathrm{~min}$ at room temperature (Human FoxP3 Buffer set BD Biosciences 560098) to fix the cells. The cells were washed and resuspended in FoxP3 buffer $\mathrm{C}$ for $30 \mathrm{~min}$ at room temperature to permeabilize the cells. The cells were then washed and resuspended in murine IgG to block nonspecific binding (10 min, room temperature) and then stained with Foxp3-Alexa 647 (BD Pharmingen 560045) for $30 \mathrm{~min}$ in the dark at room temperature. Stained cells were washed in FACS staining buffer and fixed in 1\% formalin (Polysciences) and the entire volume of cells were immediately acquired on the Fortessa cytometer (BD Biosciences). Data were analyzed with FlowJo software.

\section{Correlative studies}

\section{Nucleic acid extraction}

Formalin-fixed paraffin-embedded (FFPE) tissue sample slides were reviewed by a pathologist to confirm the presence of tumor tissue when available prior to therapy (please see schema for tissue, online supplemental figure 1); patients did not need to have tissue for correlative evaluations to be enrolled on the study. Nucleic acid isolation was performed on FFPE slides by the UNC Lineberger Comprehensive Cancer Center Translational Genomics Lab using the Maxwell 16 MDx Instrument (Promega AS3000). DNA was extracted using the Maxwell 16 FFPE Plus LEV DNA Purification Kit (Promega AS1135) following the manufacturer's protocols (Promega TM349). RNA was extracted using the Maxwell 16 LEV simplyRNA Tissue Kit (Promega AS1280) and from FFPE slides using the Maxwell 16 LEV RNA FFPE Kit (Promega AS1260) following the manufacturer's protocols (Promega TM351 and TM408, respectively). DNA and RNA quality were measured using a NanoDrop spectrophotometer (Thermo Scientific ND-2000C) and a TapeStation 4200 (Agilent G2991AA). DNA and RNA concentrations were quantified using a Qubit V.3.0 fluorometer (Life Technologies Q33216).

\section{RNA Sequencing}

Samples of total RNA extracted from FFPE tumor tissue (ROCHE High Pure FFPE kit, Indianapolis, Indiana, USA) were used to prepare Illumina TruSeq RNA Access (Cat. No. 20020189) sequencing libraries. Sequencing was performed in the UNC-Chapel Hill High Throughput
Sequencing Facility on an Illumina HiSeq 4000 platform using the Illumina HiSeq SBS 150 Cycles (PE-410-1001) with $2 \times 75$ paired end base reads.

\section{Gene expression analysis}

Paired-end FASTQ files were aligned to an Ensembl transcriptome (release 99, on reference genome GRCh38) using Star (V.2.7.3a) and transcripts quantified using Salmon (V.0.8.2). Quality of FASTQ data and quantified BAMs was verified using FastQC (V.0.11.7) and Picard's (V.1.86) CollectRnaSeqMetrics program, respectively. Expression matrices were imported into $\mathrm{R}$ using Bioconductor's tximport (V.1.10.1) prior to being upper quartile normalized and $\log 2$ transformed. Differential gene expression was calculated and compared in $\mathrm{R}$ using the DESeq2 (V.1.22.2) Bioconductor package. ${ }^{22}$ Immune gene signature expression was calculated by the mean expression of each gene within that signature.

\section{Adaptive immune receptor repertoire analysis}

Cryopreserved PBMCs were thawed and recovered in complete AIM-V medium with $10 \%$ human albumin. Cells were pelleted and lysed for RNA extraction (RNeasy Plus Mini kit, Qiagen 74134). Libraries were prepared for TCR and BCR profiling using the SMARTer Human TCR a/b Profiling Kit (Takara Bio USA, Cat. No 63516) and SMARTer Human BCR IgG IgM H/K/L Profiling Kit (Takara Bio USA, Cat. No 634467), respectively. Samples were pooled to a final concentration of $2-4 \mathrm{nM}$ before dilution of the pool to $13.5 \mathrm{pM}$ with $10 \%$ PhiX control V.3 (Ilumina, Cat. No FC-110-3001). Sequencing was performed on an Illumina MiSeq sequencer using the 600 cycle MiSeq Reagent Kit v3 (Illumina MS-102-3003) with paired-end $2 \times 300$ base pair reads.

To determine immune repertoire changes in peripheral blood during therapy, post-treatment samples were considered as the latest sample following two or more cycles of pembrolizumab. Patients receiving fewer than two cycles pembrolizumab were not assessed for this endpoint. Pretreatment samples were those collected at enrolment, or prior to, the first pembrolizumab treatment. Immune chains were inferred from FASTQ files using MiXCR (V.2.1.9) ${ }^{23}$ and paired-end reads were subject to alignment in default mode, followed by contig assembly and export. Immune repertoire similarity was calculated from the inferred chains using Horn's modified Morosita overlap index (44) (online supplemental figures 6 and 7).

\section{Whole-exome sequencing}

WES was performed on FFPE tumor tissue collected prior to treatment on this trial, with PBMCs collected serving as the matched normal. Library preparation was performed with the TruSeq DNA, PCR-Free kit (Illumina, San Diego, California, USA) and pooled samples sequenced on the HiSeq4000 platform (Illumina). Somatic and germline WES sequencing files were aligned to Hg38 using bwa (v0.7.17) and sorted, indexed, and duplicates marked 
using biobambam2 (v2.0.87). ${ }^{24}$ BAMs were realigned with Abra2 (V.2.22), followed by somatic and germline variant detection with Strelka2 (V.2.9.10), Cadabra (from Abra2 V.2.22) and Mutect2 (GATK V.4.1.4.0). ${ }^{24}$ Capture of exonic sequences was verified using the Picard (V.2.21.1) CollectHsMetrics tool, and quality of sequencing data verified using FastQC (V.0.11.8), and the Picard suite's CollectAlignmentSummaryMetrics, CollectInsertSizeMetrics, QualityScoreDistribution, and MeanQualityByCycle tools. Variants were filtered by the following criteria: proteincoding mutations only, Cadabra indel quality $>10.5$, Mutect2 indel quality $>6.8$ or single nucleotide variant (SNV) quality $>9.2$, Strelka2 indel quality $>15.2$ or SNV quality $>19.7$. Remaining variants required at least five supporting reads and a minimum read depth of 40 , or 10 supporting reads and minimum read depth of 80 if $\mathrm{MAF}<5 \%$. Variants with a MAF $>5 \%$ in normal tissue were dropped, as were variants appearing at rates above $1 \%$ in any subpopulation in either GnomAD or 1000 Genomes databases. To counter FFPE artifacts, $\mathrm{C}>\mathrm{T}$ and $\mathrm{G}>\mathrm{A}$ substitutions required a minimum MAF of $10 \%$. Tumor mutational burden (TMB) was calculated from small indels and substitutions identified by WES, and divided by the megabases adequately covered by sequencing reads. WES data were available for 26 patients.

\section{PAM50 Subtyping}

Breast tumor molecular subtyping was performed on tumor RNA-Seq data. The normalized, log2 transformed expression matrices were re-centered based on published methods $^{25}$ for comparing FFPE-derived RNA-Seq data against the microarray-derived PAM50 centroids. Samples were assigned to a subtype based on the centroid with the minimum of the absolute Spearman rank correlation coefficient. One patient had tumor available for WES but not RNASeq, and thus was assigned 'Unknown' for PAM50 subtype.

\section{Tumor imputed cell mixture}

Tumor immune cell imputation was performed from RNA-Seq data Salmon-quantified transcript counts using Cibersort $\mathrm{X}^{26}$ via the authors' website at http:/ / cibersortx. stanford.edu (online supplemental figure 8).

\section{PD-L1 Expression}

PD-L1 expression was assessed on FFPE tumor samples by Discovery Life Sciences (Newtown, PA) using the mouse anti-PD-L1 antibody clone 22C3 (QualTek), ${ }^{27}$ with membrane-specific staining of all cells (tumor and immune cells) estimated by a board-certified pathologist using a discrete scale ranging from $0 \%$ to $100 \% .{ }^{11}$ PD-L1 was considered positive if the mean proportion score was $>1 \%$ across all cells.

\section{Cytometry by time-of-flight}

We used high dimensional multiparameter mass cytometry analysis by cytometry by time-of-flight to phenotype peripheral blood lymphocytes from 16 patients profiled at 43 times across the study. The evaluation included eight responsive and eight non-responders patients after therapy. The assessments were performed longitudinally and grouped as: early (cycles 1-2), middle (cycles 3-5), late (cycles $6+$ ) treatment time points.

\section{Statistical analysis}

A sample size of 36 patients was chosen to achieve $80 \%$ power to detect a change in median PFS from 1.9 (null) to 2.9 (alternative) months at a 0.05 significance level. Assuming a 10\% dropout rate, 40 patients were enrolled. The Kaplan-Meier method was used to estimate time to event overall and for subgroups of interest, and comparisons between these subgroups were made using Fisher's exact tests to compare nominal categorical data and the Jonckheere-Terpstra method for ordinal data. Both PFS and OS were calculated from the start of treatment (C1D1). Student's t-test was used to compare effect of Cy on $\mathrm{T}_{\text {reg }}$ numbers.

Differential gene expression was compared using the default settings (Wald test) in DESeq2. ${ }^{22}$ Gene signature expression was compared using two-tailed t-test of the centered LFC values. Where indicated, $p$ values were adjusted for multiple hypothesis testing using the Benjamini-Hochberg method with $\alpha=0.20$. For TMB calculations, samples were classified as both TMB high, intermediate or low ( $\geq 20$ mutations $/ \mathrm{MB}, \geq 5$ but $<20$ mutations/ $\mathrm{MB}$, and $<5$ mutations/MB, respectively) and high or low ( $\geq 10$ mutations/MB, $<10$ mutations/MB, respectively) before comparison with the Wilcoxon rank sum test. PAM50 subtype association with clinical outcomes was assessed via Fisher's exact test. Intergroup comparisons for Morosita-Horn indices and repertoire diversity metrics (abundance, richness) were performed using the two-tailed t-test.

\section{RESULTS}

\section{Patient characteristics}

Forty patients were consented to this phase II study, of which 40 were evaluable for toxicity and survival, and 39 were evaluable for response. Patient demographics and characteristics are presented in table 1 . The majority of patients were white $(76 \%)$ with stage $0-$ III disease at diagnosis $(75 \%)$. Most patients had received prior (neo) adjuvant therapy $(\mathrm{n}=33,85 \%) ; 29(83 \%)$ underwent a prior mastectomy, $6(15 \%)$ a prior lumpectomy, and 29 (72\%) received adjuvant radiation. The median time from initial diagnosis of TNBC to first metastases for this patient population was 2 years (95\% CI 0.6 to 3.4 years). Enrolled patients had received a median of 2 prior lines of therapy for the treatment of metastatic disease (range $1-16)$, with $n=21(53 \%)$ receiving $1-3$ lines. Sites of metastatic disease included liver $(\mathrm{n}=12,30 \%)$, lung $(\mathrm{n}=14$, $35 \%)$, bone $(n=18,45 \%)$, lymph node $(n=4,10 \%)$, and brain $(n=2,5 \%)$. The majority of patients had an ECOG Performance Status of $0(n=24,60 \%)$.

\section{Toxicity and treatment discontinuation}

All 40 patients were evaluable for toxicity. Cy prior to pembrolizumab was generally well-tolerated $(n=40$, 
Table 1 Demographics and patient characteristics

\section{Patient demographics $(n=40)$}

\begin{tabular}{|c|c|}
\hline Median age, years (range) & 54.5 (33-82 years) \\
\hline \multicolumn{2}{|l|}{ Race $(n, \%)$} \\
\hline White & $28(76)$ \\
\hline Black/other & $9(24)$ \\
\hline \multicolumn{2}{|l|}{ Stage at diagnosis (n, \%) } \\
\hline $0-$-III & $30(75)$ \\
\hline IV & $10(25)$ \\
\hline $\begin{array}{l}\text { Median time to first metastases } \\
\text { (years, } 95 \% \mathrm{Cl} \text { ) }\end{array}$ & 2 (0.6 to 3.4$)$ \\
\hline \multicolumn{2}{|l|}{ Prior (neo)adjuvant therapy (n, \%) } \\
\hline Yes & $33(83)$ \\
\hline No & $7(17)$ \\
\hline \multicolumn{2}{|l|}{ Prior surgery $(\mathrm{n}, \%)$} \\
\hline Mastectomy & $29(72)$ \\
\hline Lumpectomy & $6(15)$ \\
\hline \multicolumn{2}{|c|}{ Prior radiation to breast/chest wall (n, \%) } \\
\hline Yes & $29(72)$ \\
\hline No & $11(28)$ \\
\hline \multicolumn{2}{|c|}{ Prior metastatic lines of systemic therapy (n, \%) } \\
\hline 0 to 3 & $22(58)$ \\
\hline 4 or more & $16(42)$ \\
\hline \multicolumn{2}{|l|}{ Sites of metastatic disease $(n, \%)$} \\
\hline Liver & $12(30)$ \\
\hline Lung & $14(35)$ \\
\hline Bone & $18(45)$ \\
\hline Lymph node & $4(10)$ \\
\hline Brain & $2(5)$ \\
\hline \multicolumn{2}{|l|}{ ECOG performance status (n, \%) } \\
\hline 0 & $24(60)$ \\
\hline 1 & $16(40)$ \\
\hline
\end{tabular}

figure 1A). The most common AEs were fatigue (45\%), nausea $(30 \%)$, anemia $(33 \%)$, lymphopenia $(20 \%)$, edema $(17 \%)$, and increased alanine aminotransferase $(17 \%)$. The most common grade 3 or 4 AEs were fatigue $(7 \%)$, anemia $(5 \%)$, leukopenia $(5 \%)$, neutropenia $(5 \%)$, dyspnea $(5 \%)$, diarrhea $(5 \%)$, and increased aspartate aminotransferase $(5 \%)$.

Disease progression as defined by radiographic imaging was the most common reason for treatment discontinuation $(n=25,63 \%)$. Four patients $(10 \%)$ discontinued treatment due to toxicity $(n=1$ for each of prolonged grade 2 diarrhea, elevated aspartate aminotransferase levels, pembrolizumab-induced colitis, and unspecified $\mathrm{AE}$ ). Interruptions in dosing of the pembrolizumab treatment regimen occurred in $n=11$ patients $(28 \%)$. The most common cause for interruption of treatment were grade II or greater liver toxicity $(n=5)$, grade 3 leukopenia or neutropenia $(n=2)$ and grade 2 or greater rash $(n=2)$.
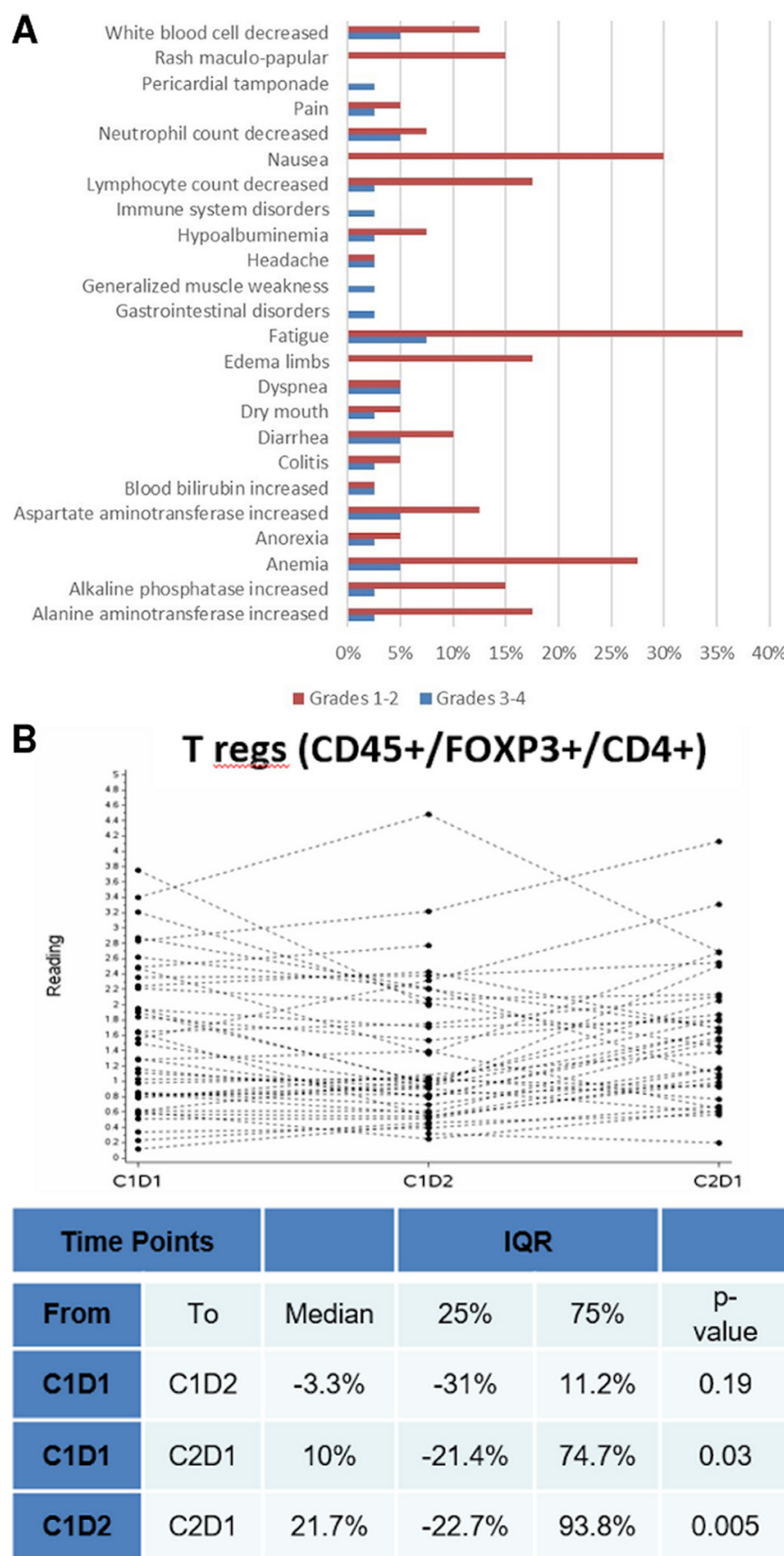

Figure 1 Adverse events and efficacy of treatment on peripheral $T_{\text {regs }}$ (A) Most common toxicities by grade (1-2 or 3-4). (B) Levels of peripheral CD45+/FOXP3+/ CD4 +regulatory T cells from blood collected on C1D1, C1D2, and C2D1, and comparison between time points.

\section{Efficacy}

Peripheral blood $\mathrm{T}_{\text {reg }}$ levels

Peripheral blood was drawn for quantification of $\mathrm{T}_{\text {regs }}$ (CD $\left.45^{+} / \mathrm{FoxP}^{+} / \mathrm{CD} 4^{+} / \mathrm{CD} 127^{-/ \mathrm{lo}}\right)$ by flow cytometry. An example of data with the gating schema used to enumerate $\mathrm{T}_{\text {regs }}$ is shown in online supplemental figure 2. There was a non-significant reduction in peripheral $\mathrm{T}_{\text {reg }}$ levels after a single dose of Cy compared with baseline (C1D1 to C1D2, median change $-3.3 \%, p=0.19$, figure $1 B$ ). However, there was a significant increase in $\mathrm{T}_{\text {regs }}$ post-CY with median $\mathrm{T}_{\mathrm{reg}}$ 

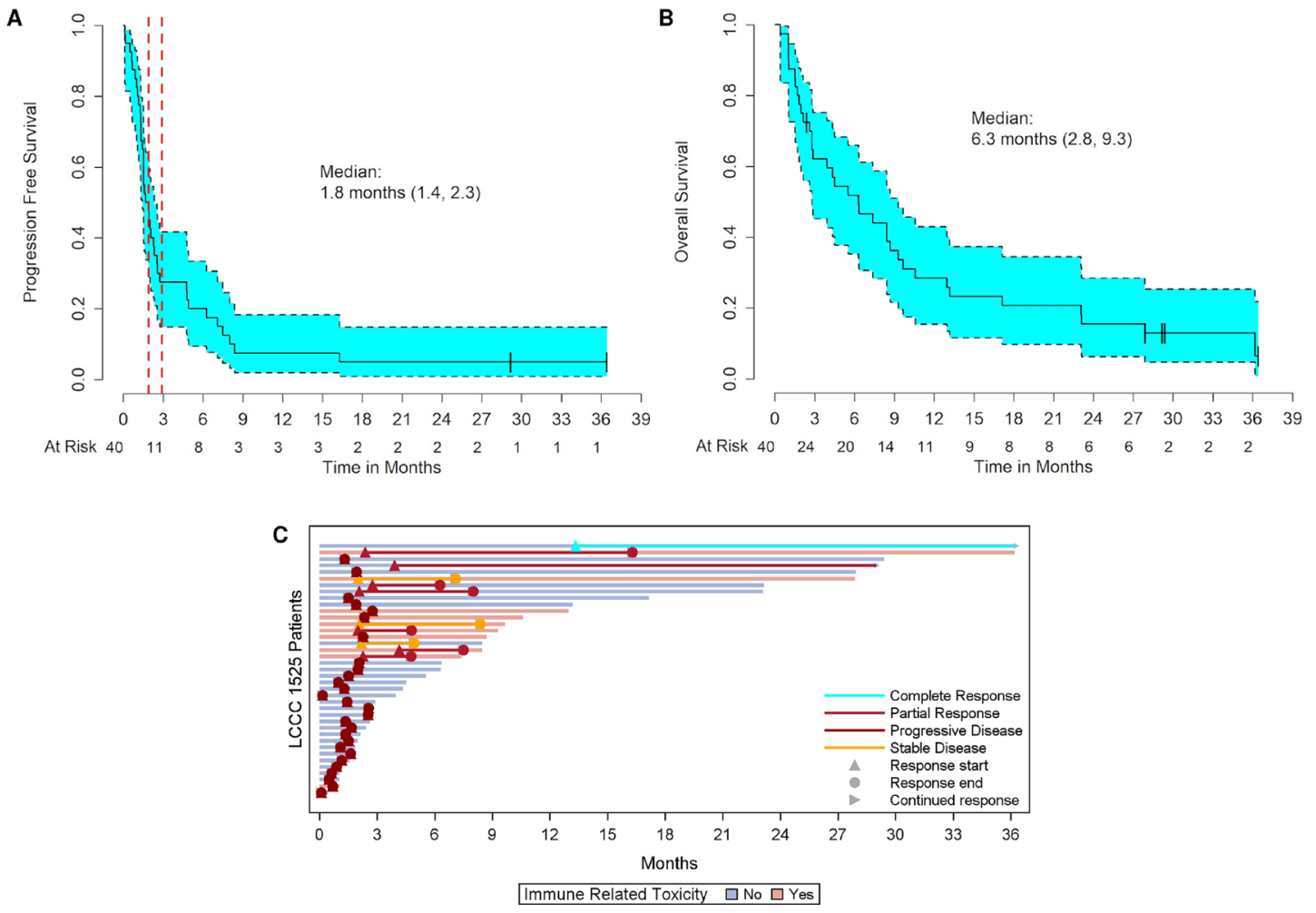

Figure 2 Survival outcomes, response rates and immune-related toxicities. Kaplan-Meier plots of (A) progression-free and (B) overall survival of patients in months, with medians and $95 \%$ Cls indicated. Vertical red dotted lines in A denote the null (1.9 months) and alternative (2.9 momths) hypotheses. $\mathrm{n}=40$ patients. (C) Swimmer's plot of patient responses (complete response, partial response, stable disease, or progressive disease) and whether patients experienced immune-related toxicities (yes/no) over time (months). Each bar represents one patient, $\mathrm{n}=39$ patients. The $\mathrm{x}$ axis represent time on treatment, starting at initiation of protocol therapy and ending when the patient dies or is censored.

numbers increasing between cycles 1 and 2 of pembrolizumab (C1D1 to C2D1, $10 \%$ increase $\mathrm{p}=0.03$; C1D2 to C2D1, 22\% increase $\mathrm{p}=0.005$; figure $1 \mathrm{~B}$ ). There was a non-significant improvement in the clinical response of patients whose $\mathrm{T}_{\text {regs }}$ decreased after CY compared with those whose $\mathrm{T}_{\text {regs }}$ increased after CY (figure 1B; $\mathrm{p}=0.08$ ).

\section{Progression-free and OS}

All 40 patients were evaluable for survival outcomes (PFS and OS). Of the 40 patients, at the time of analysis and with a median of 29 months of follow-up, $n=35$ patients $(88 \%)$ had died: $\mathrm{n}=1$ while on treatment, and $\mathrm{n}=34$ following disease progression. The median PFS was 1.8 months (95\% CI 1.4 to 2.3, figure 2A). This did not reach the prespecified alternative hypothesis of a PFS of 2.9 months, nor did median PFS significantly differ from the null hypothesis of 1.9 months. The median OS for this patient cohort was 6.3 months (95\% CI 2.8 to 9.3, figure $2 B$ ).

\section{Response and IRT}

Of the 39 patients evaluable for response, the ORR (CR +PR) was 21\% (1 CR, 7 PRs, table 2). CBR was $10 \%$ (95\% CI $2 \%$ to $24 \%$ ). An additional 3 patients had SD as best response, while the remaining 28 patients (72\%) had PD. The median DoR was 20.4 months (range 4-34 months) (figure 2C). The one patient with a CR exhibited a durable response for at least 19 months. Of the seven patients with PRs, five had a PR for at least 3 months and up to 9 months. The three patients with SD were stable for at least 3 months, with one patient experiencing stable disease for 18 months. Of the 28 patients experiencing $\mathrm{PD}, 15$ progressed within 3 months while on study, 4 more progressed by 6 months, an additional 6 progressed by 12 months, and the remaining 2 patients progressed by 18 months post-therapy.

Of the 40 patients evaluable for toxicity, 10 patients (25\%) experienced any grade IRT (table 2 ), with all but one of these patients with IRT being on therapy for more than 6 months (figure 2C). IRTs that occurred in more than two patients were rash $(n=6)$, colitis $(n=3)$ hypothyroidism $(n=3)$ and xerostomia $(n=3)$. Interestingly, the presence of an IRT correlated with response to therapy ( $p=0.02)$, while the number of prior lines of therapy and changes in $\mathrm{T}_{\text {regs }}$ did not correlate with response $(\mathrm{p} \geq 0.2)$ (table 2).

\section{Correlative objectives}

PD-L1 expression

FFPE tumors from the pretreatment primary $(n=13)$ and metastatic lesions $(n=8)$ were evaluated for PD-L1 expression in a subset of patients. There was an increased response in patients with higher percentage of tumor 
Table 2 Best objective response rates and clinical associations with response

\begin{tabular}{lrr} 
Responses & & N (total=39) \\
\hline Best objective response & 1 & 2 \\
\hline CR & 7 & 18 \\
PR & 3 & 8 \\
SD & 28 & 72 \\
PD & & P value \\
\hline Associations & Yes/no & 0.02 \\
\hline Association with best response & Yes & 0.61 \\
\hline Immune related toxicity (any grade) & No & \\
Prior lines of therapy (metastatic setting) & & 0.68 \\
Per cent change in T regs & No & 0.10 \\
C1D1 to C2D1 & No & \\
C1D1 to C1D2 & & \\
\hline
\end{tabular}

$\mathrm{CR}$, complete response; $\mathrm{PD}$, progressive disease; $\mathrm{PR}$, partial response; $\mathrm{SD}$, stable disease.

PD-L1 positivity (figure $3 \mathrm{~A}, \mathrm{~B}$ ) that did not meet the predefined definition of statistical significance using thresholds for positivity of $1 \%$ or $10 \%$ (Wilcoxon rank sum $\mathrm{p}>0.37$, data not shown).

\section{Gene expression, WES, and tumor mutational burden}

To evaluate for biomarkers of response to therapy with Cy/pembrolizumab, we performed bulk RNA-seq on tumor tissue prior to therapy. Gene expression differences were observed in all samples from responders $(\mathrm{CR}+\mathrm{PR})$ versus non-responders $(\mathrm{SD}+\mathrm{PD})$ treated with Cy/pembrolizumab, with ANKRD30A, SYCE1, and TUBB2B being higher in responders while KRT1, NRAP, and KRT2 were higher in non-responders (figure 3C, online supplemental figure 3 ).

WES demonstrated that TP53 was the most frequently mutated gene in pretreatment tumors $(n=14 / 26,54 \%$ of patients) (figure 3D). The three patients with identified BRCA2 mutations did not benefit from therapy as they experienced PD as best response (figure 3D). To assess the intrinsic subtype of the tumors treated in this study, PAM50 molecular subtyping was performed on all samples. The majority of the tumors were the basal-like subtype, with no association between predicted subtype and either response or CBR (Fisher's exact test $p>0.29$, figure 3C). There was no association between TMB and either CB or response (Wilcoxon rank sum test $p>0.288$, online supplemental figure $4 A, B)$. This may be due to the paucity of patients with high TMB as the patient who had a CR to Cy/pembrolizumab had a high TMB of greater than 20 mutations per sequenced megabase (figure 3D, data not shown). Similarly, there was no association between tumor PD-L1 expression and TMB (Wilcoxon rank sum test $\mathrm{p}>0.171$ ) using PD-L1 positivity thresholds of either $1 \%$ or $10 \%$. Gene set enrichment analysis and immune gene signatures did not reveal any significant associations with either $\mathrm{CB}$ or response (online supplemental figure
5A,B). No somatic mutations were associated with clinical response in this study. The mutation profile of the study cohort did include multiple uncommon mutations that have been associated with immunosuppressive tumor immune microenvironment features, including dampening of interferon responses and decreased $\mathrm{T}$ cell infiltration (ARID1A, PIK3CA, BAP1) in tumors other than breast cancer. ${ }^{28-30}$

\section{Adaptive immune receptor repertoire diversity}

To assess how adaptive immune receptor repertoires may relate to ICI response, we performed T and B cell receptor repertoire profiling of pretreatment tumor samples and PBMCs. Specifically, we investigated several diversity metrics associated with $\mathrm{T}$ cell receptor alpha (TRA) and beta (TRB) chains and $\mathrm{B}$ cell receptor immunoglobulin lambda (IgL), kappa (IgK) and heavy $(\operatorname{IgH})$ chains. For each sample, we evaluated diversity indices that represent the number of unique clonotypes (eg, richness, Chao1), the relative frequencies of each clonotype (eg, Shannon entropy, evenness), and the total number of all clonotypes (abundance). Overall, there were no significant differences in either the pretreatment tumor, pretreatment peripheral, post-treatment (defined as after two or more cycles of pembrolizumab) peripheral, or pretreatment versus post-treatment peripheral repertoire diversity measures across IgH, IgK, IgL, TRB or TRA chains by either CB or response (data not shown). We noted a small but significant association when examining $\mathrm{T}$ cell TRB chain similarity in an intra-group fashion, indicating that patients with CBR (online supplemental figure 4C) or response (online supplemental figure 4D) had less overlap in their detected TRB chains than those with progression or non-response. However, these differences were not significant after correction for multiple comparisons. Patients with CBR or clinical response to therapy were also more likely to have a preserved peripheral TRB 
A

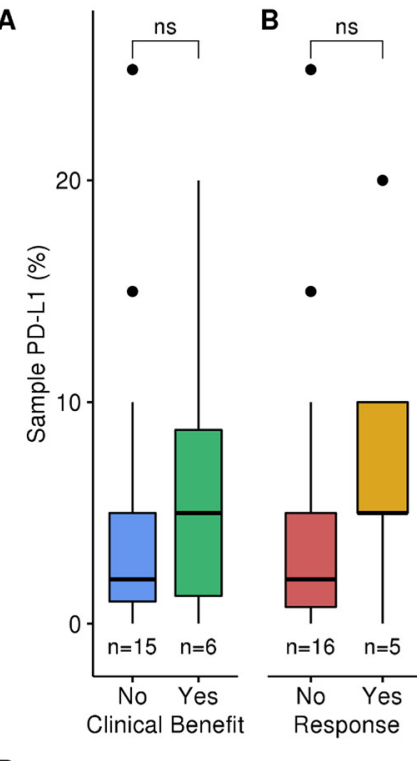

C

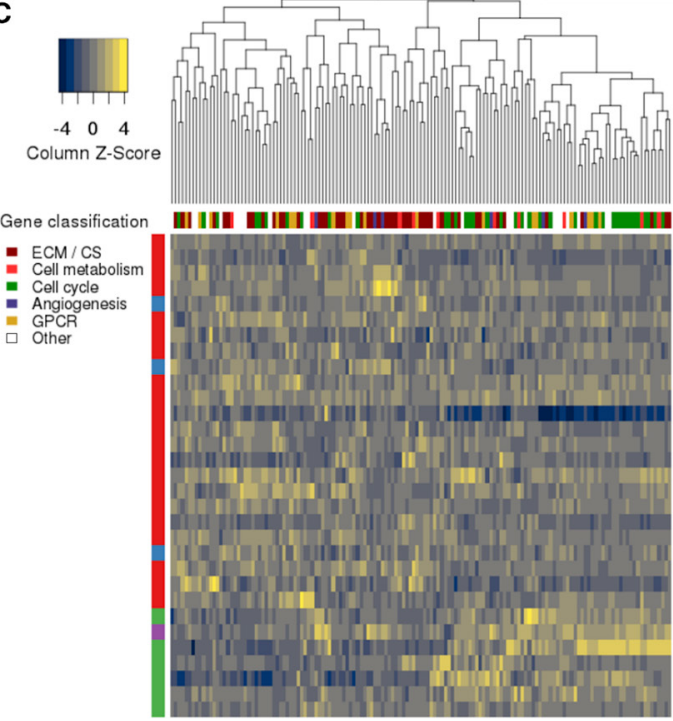

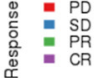

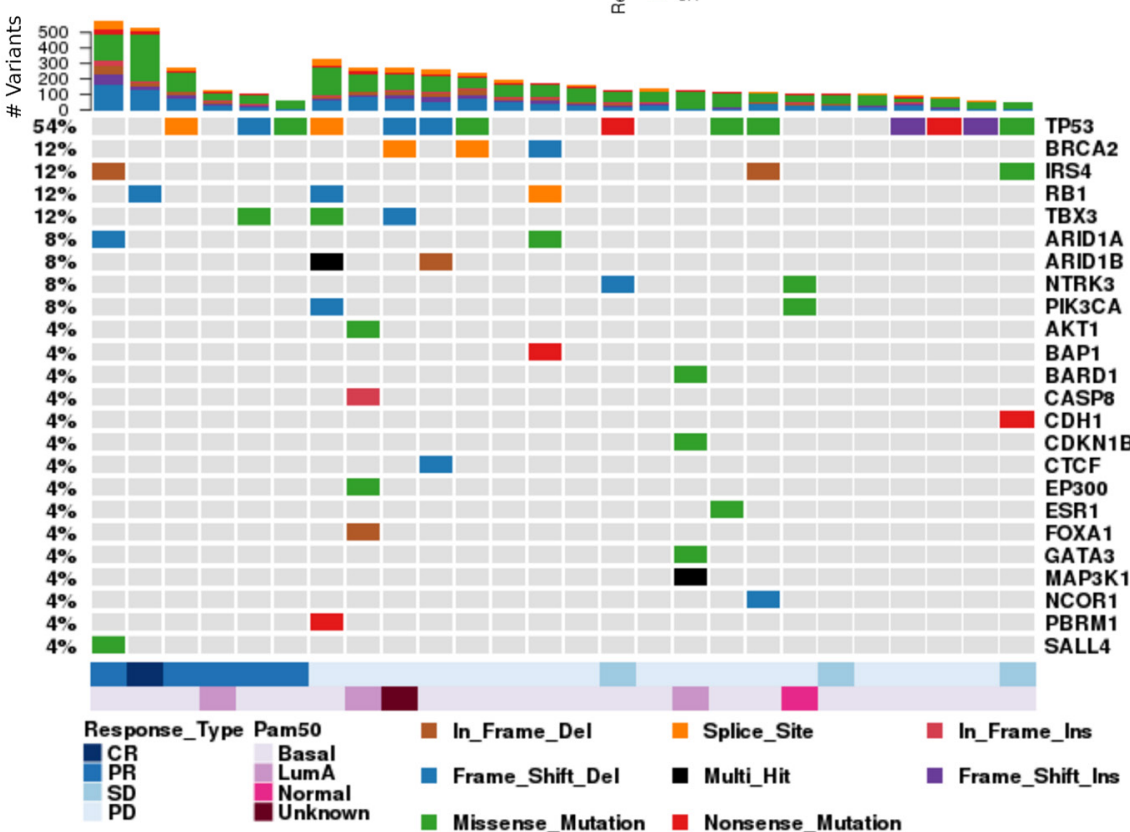

Figure 3 Tumor genomic and immune features. (A, B) Tumor PD-L1 expression was not significantly associated with either clinical benefit (CR+PR+SD; A) or response (CR+PR; B) to therapy by t-test (shown), or by stratifying PD-L1 positive/negative (PD-L1 <1\% vs $\geq 1 \%$, or PD-L1 <10\% vs $\geq 10 \%$; Wilcoxon rank-sum test, not shown). (C) Differential gene expression ( $\alpha<0.2)$ in archival tumor samples, by treatment response (CR+PR; bottom rows) vs non-response (SD+PD; top rows). (D) Frequently somatically mutated genes implicated in breast cancer per COSMIC Tier one classification by variant type. Raw tumor mutational burden is noted at the top of each sample column. Treatment response and tumor PAM50 subtype for each sample is listed at bottom of each column. CR, complete response; ns, not significant; PD, progressive disease; PR, partial response; $\mathrm{SD}$, stable disease.

chain repertoire after two or more cycles of pembrolizumab (online supplemental figure 4E,F). We noted small differences between tumor IGH chain abundance (raw number of chains, online supplemental figure $4 \mathrm{G}, \mathrm{H}$ ) and richness (online supplemental figure $4 \mathrm{I}, \mathrm{J}$ ) in patients who went on to have $\mathrm{CB}$ or response to therapy.

To better explore the relationships between adaptive immune receptor repertoire features and clinical outcomes, univariable Cox regression models were fit with PFS as the response variable and repertoire diversity metrics in pretreatment blood and among those who received at least two cycles pembrolizumab as the predictor variables. We found a significant association (false discovery rate (FDR) $\alpha<0.2$ ) with multiple B cell diversity features (IGL abundance, IGH Shannon entropy, evenness, and abundance) in post-treatment peripheral blood samples (figure 4A), with directions similar to associations in the pretreatment tumor and 
A

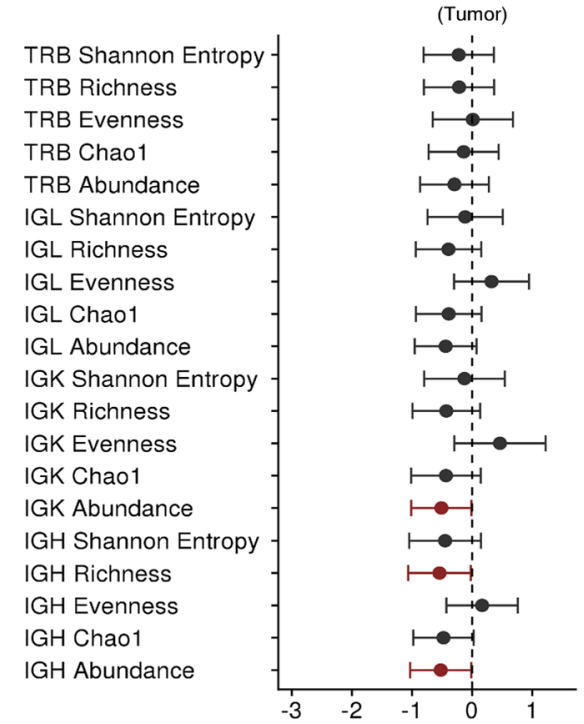

B

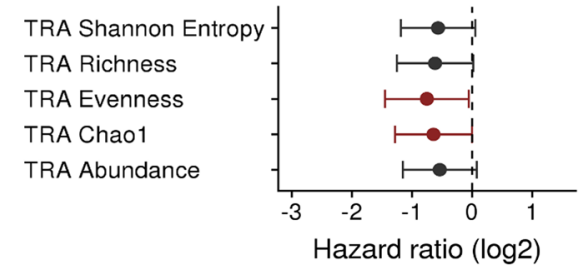

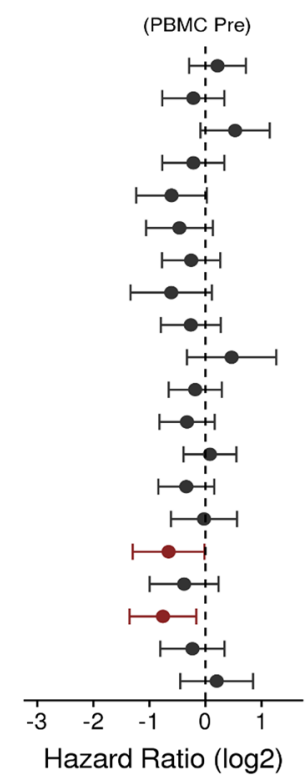

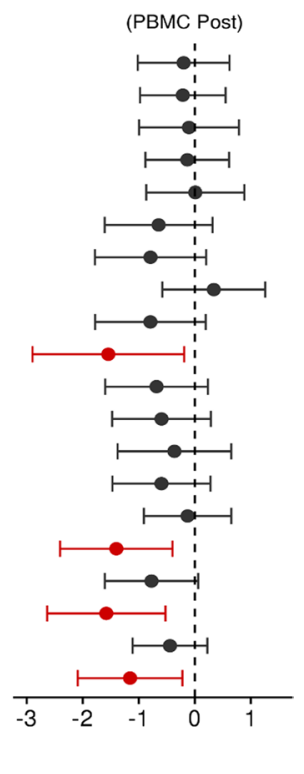

Hazard Ratio $(\log 2)$

Figure 4 Immune repertoire diversity. (A-D) Univariable Cox proportional hazards models for PFS showing adaptive immune receptor repertoire diversity measures derived from pre-treatment tumor RNA-Seq (A), PBMC-derived amplicon sequencing prepembrolizumab (B), and PBMC-derived amplicon sequencing post-pembrolizumab (after at least 2 cycles of pembrolizumab) (C). For readability, TRA chain metrics from pretreatment tumor RNA-Seq are displayed in (D). Bright red indicates measures which were significant after FDR adjustment at $\alpha<0.20$, dark red at $p<0.05$. FDR, false discovery rate; PBMC, peripheral blood mononuclear cell; PFS, progression-free survival.

peripheral B cell repertoires (figure 4A). TRA evenness and Chao1 in pretreatment tumor samples also demonstrated a less robust $(\mathrm{p}<0.05)$ association with clinical outcomes (figure 4B).

\section{Non-nodal tumor tissue subgroup}

Gene set enrichment analysis using on RNA sequencing on a subset of tumors $(n=22$, online supplemental figure 6) showed CBR in patients receiving Cy/pembrolizumab therapy was associated with several immune pathways including the CXCR4, BCR, IL-2, NO2-IL12 pathways and IL-2 signaling. Similarly, immune gene signatures demonstrated upregulation in B cell immune gene signatures correlated with CBR. We hypothesized that B cell gene expression may be biased by samples identified as lymph nodes at the time of surgery, and performed a subgroup analysis that would exclude tumor-containing lymph nodes $(n=6)$.

In this exploratory analysis, we noted several associations between immune features and clinical outcomes in non-nodal tumor tissues $(\mathrm{n}=25)$, though none remained significant after multiple testing correction (online supplemental figure 7). There was a significant correlation between $\mathrm{CB}$ (online supplemental figure 7A) and published mRNA signatures for CD56-dim NK cells, ${ }^{31}$ and claudin-low intrinsic subtype. ${ }^{32}$ Response to immune therapy (online supplemental figure 7B) was significantly correlated with published mRNA signatures for $\mathrm{IgG}^{33}$ three B cell signatures ${ }^{31} 3435$ and CD56-dim NK cells. ${ }^{31}$

\section{Leucocyte phenotype abundance in the TME}

The heterogeneity of the TME can be assessed from RNA-seq data using informatics approaches to estimate the abundance of the immune cells present. ${ }^{36}$ Estimated cell mixture abundances using CibersortX did not reveal any significant differences between patient groups by $\mathrm{CB}$ or response for the detected cell subpopulations (online supplemental figure 8). Recently our group identified six different immune subtypes present across all TCGA tumors. Using this approach ${ }^{37}$ the RNA expression from all tumor samples was strongly representative of the C4 'lymphocyte depleted' immune subtype.

\section{Abundance and phenotype of peripheral lymphocytes}

To confirm our tumor findings, we performed mass cytometry on peripheral blood from 8 patients who responded to therapy and compared this to 8 patients who did not respond to treatment. We observed a significant increase in CD45 +CD14-, HLA-DR+, CD20+, CD19+, IgD-, CD38+, $\mathrm{CD} 27$ +plasmablasts in responders when compared with non-responders only after Cycles 1-2 of treatment (online supplemental figure 9). There was no difference 
found in later cycles of therapy. Although we did not note differences in the frequency of total CD4 +or CD8+T cells in the peripheral blood at any time during treatment, we observed increased expression of PD-1, TIM-3, and CD25 on responder $\mathrm{CD} 8+\mathrm{T}$ cells when isolated only during cycles 1-2 post-therapy (online supplemental figure 10). Thus, these data confirm our findings regarding activation of antibody-secreting cells after therapy and additionally demonstrate an increase in activation markers in peripheral blood CD8 $+\mathrm{T}$ cells post-therapy.

\section{DISCUSSION}

While ICI has changed the landscape of patient care for multiple solid tumors, there is still significant room for improvement to increase response rate and durability of response, particularly in breast cancer. As the presence of $\mathrm{T}_{\text {reg }}$ cells in tumors has been shown to reduce response to ICI, we hypothesized in this multicenter, phase II trial that a single, low dose of Cy prior to the PD-1 inhibitor pembrolizumab would increase PFS in patients with mTNBC compared with historical controls. The PFS of 1.8 months failed to confirm this hypothesis. The ORR to Cy prior to pembrolizumab was $21 \%$, similar to single agent PD-1 inhibition alone in prior studies. ${ }^{11}$ Clinical response did correlate with the presence of immunerelated toxicities. Interestingly, we found that response to $\mathrm{Cy} /$ pembrolizumab therapy correlated with enhanced expression of B cell metagene signatures in pretreatment tumors and BCR diversity in peripheral blood, mirroring previous preclinical work ${ }^{34}$ that indicated a critical role for antibody production by $\mathrm{B}$ cells and $\mathrm{T}$ cell help in the response to dual ICI.

Approaches to deplete $T_{\text {regs }}$ have been challenging due to the lack of selectable markers that are present on $\mathrm{T}_{\text {regs }}$. We chose to use low-dose Cy since this approach illustrated benefit in our prior murine studies, and Cy is a commonly prescribed chemotherapy for breast cancer. ${ }^{19}$ The overall impact of low-dose CY was quite modest with a median decrease of $3.3 \%$ in peripheral blood $\mathrm{T}_{\text {regs }} 1$ day after administration, which did not meet prespecified thresholds for significance. Cy was not continued after the initial priming dose, as continued exposure to Cy diminished the effector $\mathrm{T}$ cell response to breast cancer in animal models. ${ }^{38}$ Unfortunately, perhaps due to lymphopeniadriven expansion, there was a marked increase in peripheral blood $\mathrm{T}_{\text {regs }}$ after discontinuation of $\mathrm{CY}$ with a median $10 \%$ increase between receipt of CY and the initiation of cycle 2 of therapy. The Nivolumab After Induction Treatment in Triple-negative Breast Cancer Patients (TONIC) study in mTNBC similarly demonstrated that short exposure to Cy does not improve durvalumab response. ${ }^{5}$

Here, we found a correlation with CBR and IRT. While toxicities are commonly associated with anti-PD-1 monoclonal antibody therapy, this has not routinely correlated with response in other studies. In KEYNOTE-012B, ${ }^{11} 16 \%$ of patients had grade III or greater AEs, and this was not correlated with response. KEYNOTE-086 $6^{21} 39$ evaluated pembrolizumab monotherapy in a group of 170 patients with $\mathrm{mTNBC}$ who had progressed following previous therapy and were enrolled independent of the expression of PD-L1, as was done in the current trial. Treatmentrelated AEs were seen in $60 \%$ of enrolled patients, with $12 \%$ having grade III or greater AEs; the presence of significant AEs was not associated with overall outcome. Future studies that target $\mathrm{T}_{\text {reg }}$ depletion with ICI should determine if clinical efficacy correlates with IRE in this setting.

The initial hypothesis for this study was that depletion of $\mathrm{T}_{\text {regs }}$ would enhance the antitumor activity of $\mathrm{CD}^{+} \mathrm{T}$ cells in the TME. Thus, we were surprised that increased $\mathrm{B}$ cell signature expression prior to therapy best correlated with response. This result was confirmed by using several different B cell signatures. Additionally, there was a correlation between measures of B cell receptor repertoire diversity in the peripheral blood and response to therapy. We assessed multiple indices of diversity in this study, including species richness, evenness, and Shannon entropy, an index that combines richness and evenness. A correlation was found with increased abundance and richness of Ig heavy and light chain genes in the peripheral blood, suggesting that B cell population diversity correlated with response. Unfortunately, we were not able to evaluate the TME during therapy to determine if these correlations were also found in the tumor. It is currently not clear what effects anti-PD-1 antibody therapy has on B cell responses and whether these mediate CBR. Activated B cells express PD-1, which limits B cell proliferation and antibody production. ${ }^{40}$ Our previous murine data indicated a critical role for antibody generation by $\mathrm{B}$ cells in their function after ICI, and it is intriguing to hypothesize that autoantibody generation may be an important part of the immune response to ICI. ${ }^{34}$

Using RNA-seq from tumors prior to therapy, we evaluated if the expression of specific pathways was associated with response to $\mathrm{Cy} /$ pembrolizumab. Multiple pathways were associated with CBR including genes associated with caspase activation (apoptosis), the NFAT pathway and C-MYC repression. Granzyme activity, which is upstream of caspase activation and apoptosis and a mechanism of cytotoxicity by NK and T cells, was associated with response. Repression of MYC activity is associated with the expression of integrins, chemokines and anti-apoptotic proteins that may be critical to the response to $\mathrm{Cy} /$ pembrolizumab. Finally, when all tumors including LNs were analyzed, a number of immune relevant pathways were associated with CBR including major histocompatibility complex (MHC) class II antigen expression critical for the activation of $\mathrm{CD} 4^{+} \mathrm{T}$ cells. However, these analyses were not significant when corrected for multiple testing using an FDR alpha $<0.2$, but these data may inform future studies.

In summary, we found that low-dose Cy was not effective in depleting $\mathrm{T}_{\text {regs }}$ prior to anti-PD1 therapy. This combination did not meet the prespecified 1 month increase in PFS that the trial was designed to detect nor 
lead to statistically significant decreased peripheral blood $\mathrm{T}_{\text {regs }}$. CBR and response were correlated with immune toxicities, which were, in general, modest. Somewhat surprisingly, but in agreement with our preclinical data, we found that CBR was most associated with expression of B cell gene signatures in pretreatment tumor specimens. These data suggest that the B cell response may be important in the immune response to ICI therapy in patients with $\mathrm{mTNBC}$, as previously suggested in preclinical studies. ${ }^{34}$

\section{Author affiliations}

${ }^{1}$ Duke Cancer Institute, Durham, North Carolina, USA

${ }^{2}$ Division of Medical Oncology, University of North Carolina School of Medicine, Chapel Hill, North Carolina, USA

${ }^{3}$ Lineberger Comprehensive Cancer Center, University of North Carolina School of Medicine, Chapel Hill, North Carolina, USA

${ }^{4}$ Division of Microbiology and Immunology, University of North Carolina School of Medicine, Chapel Hill, North Carolina, USA

${ }^{5}$ Department of Pathology and Laboratory Medicine, University of North Carolina School of Medicine, Chapel Hill, North Carolina, USA

${ }^{6}$ Department of Hematology and Oncology, George Washington Cancer Center, Washington, District of Columbia, USA

${ }^{7}$ Division of Hematology/Oncology, University of Pittsburgh, University of Pittsburgh, Pittsburgh, Pennsylvania, USA

${ }^{8}$ Abramson Cancer Center, University of Pennsylvania Perelman School of Medicine, Philadelphia, Pennsylvania, USA

${ }^{9}$ Division of Hematology/Oncology, Cone Health Cancer Center, Greensboro, North Carolina, USA

${ }^{10}$ Rex Hematology Oncology Associates, Rex Cancer Care, Raleigh, North Carolina, USA

${ }^{11}$ Department of Genetics, University of North Carolina School of Medicine, Chapel Hill, North Carolina, USA

${ }^{12}$ Division of Hematology, University of North Carolina School of Medicine, Chapel Hill, North Carolina, USA

Twitter Amanda E D Van Swearingen @DrVanSwearingen and Luz A Cuaboy @ Icuaboy 14

Acknowledgements The authors thank the following groups at UNC-Chapel Hill for their assistance with this study: Office of Clinical and Translational Research (OCTR), Immune Monitoring and Genomics Facility (IMGF), High Throughput Sequencing Facility (HTSF), the LCCC Clinical Protocol Office (CP0), UNC-Lineberger Bioinformatics Core ( $\mathrm{LBC})$ and the UNC Flow Cytometry Facility and Marie lannone for assistance with mass cytometry experiments. OCTR, IMGF, HTSF, CPO and LBC are supported by P30 CA016086. We also thank the patients in this study and their families, without whom this study would not have been possible.

Contributors Conception and design: CKA, BGV and JSS. Development of methodology: CKA, CP, BGV and JSS. Acquisition of data (provided animals, acquired and managed patients, provided facilities, etc): CKA, MJS, LAC, ECD, TJ, HM, KR-H, 00 , VG, RJ and RK. Analysis and interpretation of data (eg, statistical analysis, biostatistics, computational analysis): CKA, AEDVS, BGV, MGW, DTM and ADW. Writing, review, and/or revision of the manuscript: CKA, AEDVS, MW, DTM, ECD, BGV, CP, JSS and LC. Administrative, technical, or material support (ie, reporting or organizing data, constructing databases): CKA, AEDVS, MGW, DTM, LAC, ALG. Study supervision: CKA, ECD, BV and JSS. JSS is the guarantor for the study.

Funding Merck Sharp \& Dohme, a subsidiary of Merck \& Co, Kenilworth, NJ, USA (MSD) provided financial support for the study. This work as also supported by CKA: Translating Duke Health Susan G. Komen for the Cure (BV), V Foundation for Cancer Research (CKA and BV), University Cancer Research Fund (JS) and P50 CA058223 (CP).

Competing interests CKA receives research funding from PUMA, Lilly, MSD, Seattle Genetics, Nektar, Tesaro, and G1 Therapeutics, ZION, Novartis, Pfizer; compensation for consulting from Genentech, Eisai, IPSEN, Seattle Genetics, AstraZeneca, Novartis; and royalties from UpToDate and Jones and Bartlett. BV holds equity in GeneCentric Therapeutics. $\mathrm{CP}$ is an equity stockholder and consultant of BioClassifier, and an equity stock holder, consultant, and Board of
Directors member of GeneCentric Therapeutics. CP is also listed as an inventor on patent applications for the Breast PAM50 assay. JS receives funding from MSD, GSK, and Carisma, is a scientific consultant for PIQUE Therapeutics and has filed IP for the use of STING agonists to enhance CAR T cell for breast cancer. The other authors have no conflicts requiring disclosure.

Patient consent for publication Not applicable.

Ethics approval This study was approved by UNC IRB \#16-1025.

Provenance and peer review Not commissioned; externally peer reviewed.

Data availability statement Genomics data are available in a public open access repository. All other data relevant to the study are included in the article or uploaded as supplementary information.

Supplemental material This content has been supplied by the author(s). It has not been vetted by BMJ Publishing Group Limited (BMJ) and may not have been peer-reviewed. Any opinions or recommendations discussed are solely those of the author(s) and are not endorsed by BMJ. BMJ disclaims all liability and responsibility arising from any reliance placed on the content. Where the content includes any translated material, BMJ does not warrant the accuracy and reliability of the translations (including but not limited to local regulations, clinical guidelines, terminology, drug names and drug dosages), and is not responsible for any error and/or omissions arising from translation and adaptation or otherwise.

Open access This is an open access article distributed in accordance with the Creative Commons Attribution Non Commercial (CC BY-NC 4.0) license, which permits others to distribute, remix, adapt, build upon this work non-commercially, and license their derivative works on different terms, provided the original work is properly cited, appropriate credit is given, any changes made indicated, and the use is non-commercial. See http://creativecommons.org/licenses/by-nc/4.0/.

\section{ORCID iDs}

Mark G Woodcock http://orcid.org/0000-0001-7348-4980

Amanda E D Van Swearingen http://orcid.org/0000-0002-4287-9741

\section{REFERENCES}

1 Dent R, Trudeau M, Pritchard Kl, et al. Triple-negative breast cancer: clinical features and patterns of recurrence. Clin Cancer Res 2007;13:4429-34.

2 Carey L. Old drugs, new tricks for triple-negative breast cancer. Lancet Oncol 2015;16:357-9.

3 Prat A, Adamo B, Cheang MCU, et al. Molecular characterization of basal-like and non-basal-like triple-negative breast cancer. Oncologist 2013;18:123-33.

4 Sharma P. Biology and management of patients with triple-negative breast cancer. Oncologist 2016;21:1050-62.

5 Voorwerk L, Slagter M, Horlings HM, et al. Immune induction strategies in metastatic triple-negative breast cancer to enhance the sensitivity to PD-1 blockade: the TONIC trial. Nat Med 2019;25:920-8.

6 Harrell JC, Prat A, Parker JS, et al. Genomic analysis identifies unique signatures predictive of brain, lung, and liver relapse. Breast Cancer Res Treat 2012;132:523-35.

7 Tutt A, Tovey H, Cheang MCU, et al. Carboplatin in BRCA1/2mutated and triple-negative breast cancer BRCAness subgroups: the TNT trial. Nat Med 2018;24:628-37.

8 Schmid P, Adams S, Rugo HS, et al. Atezolizumab and nabpaclitaxel in advanced triple-negative breast cancer. $N$ Engl J Med 2018;379:2108-21.

9 Sambade MJ, Prince G, Deal AM, et al. Examination and prognostic implications of the unique microenvironment of breast cancer brain metastases. Breast Cancer Res Treat 2019;176:321-8.

10 Sammons S, Van Swearingen AED, Anders CK. The promise of immunotherapy for breast cancer brain metastases. Curr Breast Cancer Rep 2019;11:241-7.

11 Nanda R, Chow LQM, Dees EC, et al. Pembrolizumab in patients with advanced triple-negative breast cancer: phase lb KEYNOTE-012 study. J Clin Oncol 2016;34:2460-7.

12 Cortes J, Cescon DW, Rugo HS, et al. Pembrolizumab plus chemotherapy versus placebo plus chemotherapy for previously untreated locally recurrent inoperable or metastatic triple-negative breast cancer (KEYNOTE-355): a randomised, placebo-controlled, double-blind, phase 3 clinical trial. Lancet 2020;396:1817-28.

13 Bohling SD, Allison $\mathrm{KH}$. Immunosuppressive regulatory T cells are associated with aggressive breast cancer phenotypes: a potential therapeutic target. Mod Pathol 2008;21:1527-32. 
14 Liu S, Foulkes WD, Leung S, et al. Prognostic significance of FOXP3+ tumor-infiltrating lymphocytes in breast cancer depends on estrogen receptor and human epidermal growth factor receptor-2 expression status and concurrent cytotoxic T-cell infiltration. Breast Cancer Res 2014;16:432.

15 Perez SA, Karamouzis MV, Skarlos DV, et al. CD4+CD25+ regulatory T-cell frequency in HER-2/neu (HER)-positive and HERnegative advanced-stage breast cancer patients. Clin Cancer Res 2007;13:2714-21.

16 Danilin S, Merkel AR, Johnson JR, et al. Myeloid-derived suppressor cells expand during breast cancer progression and promote tumorinduced bone destruction. Oncoimmunology 2012:1:1484-94.

17 Bergenfelz C, Roxå A, Mehmeti M, et al. Clinical relevance of systemic monocytic-MDSCs in patients with metastatic breast cancer. Cancer Immunol Immunother 2020;69:435-48.

18 Honkanen TJ, Tikkanen A, Karihtala P, et al. Prognostic and predictive role of tumour-associated macrophages in HER2 positive breast cancer. Sci Rep 2019;9:10961.

19 Taylor NA, Vick SC, Iglesia MD, et al. Treg depletion potentiates checkpoint inhibition in claudin-low breast cancer. J Clin Invest 2017; 127:3472-83.

20 Eisenhauer EA, Therasse P, Bogaerts J, et al. New response evaluation criteria in solid tumours: revised RECIST guideline (version 1.1). Eur J Cancer 2009;45:228-47.

21 Adams S, Schmid P, Rugo HS, et al. Pembrolizumab monotherapy for previously treated metastatic triple-negative breast cancer: cohort a of the phase II KEYNOTE-086 study. Ann Oncol 2019;30:397-404.

22 Love MI, Huber W, Anders S. Moderated estimation of fold change and dispersion for RNA-seq data with DESeq2. Genome Biol 2014;15:550.

23 Bolotin DA, Poslavsky S, Mitrophanov I, et al. MiXCR: software for comprehensive adaptive immunity profiling. Nat Methods 2015;12:380-1.

24 Bartha Áron, Győrffy B. Comprehensive outline of whole exome sequencing data analysis tools available in clinical oncology. Cancers 2019;11 doi:10.3390/cancers11111725

25 Picornell AC, Echavarria I, Alvarez E, et al. Breast cancer PAM50 signature: correlation and concordance between RNA-seq and digital multiplexed gene expression technologies in a triple negative breast cancer series. BMC Genomics 2019;20:452.

26 Newman AM, Steen CB, Liu CL, et al. Determining cell type abundance and expression from bulk tissues with digital cytometry. Nat Biotechnol 2019;37:773-82

27 Dolled-Filhart M, Locke D, Murphy T, et al. Development of a prototype immunohistochemistry assay to measure programmed death ligand-1 expression in tumor tissue. Arch Pathol Lab Med 2016;140:1259-66.

28 Borcoman E, De La Rochere P, Richer W, et al. Inhibition of PI3K pathway increases immune infiltrate in muscle-invasive bladder cancer. Oncoimmunology 2019;8:e1581556.

29 Figueiredo CR, Kalirai H, Sacco JJ, et al. Loss of BAP1 expression is associated with an immunosuppressive microenvironment in uveal melanoma, with implications for immunotherapy development. $J$ Pathol 2020;250:420-39.

$30 \mathrm{Li}$ J, Wang W, Zhang Y, et al. Epigenetic driver mutations in ARID1A shape cancer immune phenotype and immunotherapy. J Clin Invest 2020;130:2712-26.

31 Bindea G, Mlecnik B, Tosolini M, et al. Spatiotemporal dynamics of intratumoral immune cells reveal the immune landscape in human cancer. Immunity 2013;39:782-95.

32 Prat A, Parker JS, Karginova O, et al. Phenotypic and molecular characterization of the claudin-low intrinsic subtype of breast cancer. Breast Cancer Res 2010;12:R68.

33 Fan C, Prat A, Parker JS, et al. Building prognostic models for breast cancer patients using clinical variables and hundreds of gene expression signatures. BMC Med Genomics 2011;4:3.

34 Hollern DP, Xu N, Thennavan A, et al. B cells and T follicular helper cells mediate response to checkpoint inhibitors in high mutation burden mouse models of breast cancer. Cell 2019;179:1191-206.

35 Iglesia MD, Vincent BG, Parker JS, et al. Prognostic B-cell signatures using mRNA-seq in patients with subtype-specific breast and ovarian cancer. Clin Cancer Res 2014;20:3818-29.

36 Newman AM, Liu CL, Green MR, et al. Robust enumeration of cell subsets from tissue expression profiles. Nat Methods 2015;12:453-7.

37 Thorsson V, Gibbs DL, Brown SD, et al. The immune landscape of cancer. Immunity 2018;48:812-30.

38 Machiels JP, Reilly RT, Emens LA, et al. Cyclophosphamide, doxorubicin, and paclitaxel enhance the antitumor immune response of granulocyte/macrophage-colony stimulating factor-secreting whole-cell vaccines in HER-2/neu tolerized mice. Cancer Res 2001;61:3689-97.

39 Adams S, Loi S, Toppmeyer D, et al. Pembrolizumab monotherapy for previously untreated, PD-L1-positive, metastatic triple-negative breast cancer: cohort B of the phase II KEYNOTE-086 study. Ann Oncol 2019;30:405-11.

40 Okazaki T, Maeda A, Nishimura H, et al. PD-1 immunoreceptor inhibits $B$ cell receptor-mediated signaling by recruiting Src homology 2-domain-containing tyrosine phosphatase 2 to phosphotyrosine. Proc Natl Acad Sci U S A 2001;98:13866-71. 